\title{
Effect of Age in Second Language Acquisition: A Critical Review from the Perspective of Critical Period Hypothesis and Ultimate Attainment
}

\author{
Mohammad Mosiur Rahman ${ }^{1}$, Ambigapathy Pandian ${ }^{1}$, Abdul Karim² \& Faheem Hasan Shahed ${ }^{3}$ \\ ${ }^{1}$ School of Languages, Literacies and Translation, Universiti Sains Malaysia, Penang, Malaysia \\ ${ }^{2}$ School of Educational Studies, Universiti Sains Malaysia, Penang, Malaysia \\ ${ }^{3}$ BRAC Institute of Languages, BRAC University, Dhaka, Bangladesh \\ Correspondence: Mohammad Mosiur Rahman, School of Languages, Literacies and Translation, Universiti Sains \\ Malaysia, Penang, Malaysia. E-mail: mosiurbhai@gmail.com
}

Received: April 26, 2017 Accepted: June 30, 2017 Online Published: July 16, 2017

doi:10.5539/ijel.v7n5p1 URL: http://doi.org/10.5539/ijel.v7n5p1

\begin{abstract}
This article addresses the effect of age in Second Language Acquisition (SLA), posing the relative question that whether the Critical Period hypothesis (CPH) exists in Second Language (SL), and if existing, how it is associated duly with SLA. The justification of comparing the achievement of L1 and L2 learners on the basis of Ultimate Attainment (UA) in the establishment of Critical Period Hypothesis, is also discussed. In the methodology, secondary data analysis was used to answer of research questions. To achieve a reliable result from the wide range of secondary data primarily from journal articles, a systemic search has been adopted. In conclusion, compare and contrast was made with earlier studies to show the findings of the study and to scope future research.
\end{abstract}

Keywords: SLA, Bi-lingualism, Critical Period hypothesis, ultimate attainment

\section{Introduction}

Second language acquisition (SLA) increasingly generates pragmatic debates in the broader field of psycholinguistics. Considering the importance and differences with language acquisition in L1 and L2, SLA flourished as a distinct paradigm for research and has become a widely taught subject in graduate and postgraduate programs worldwide (Rahman \& Pandian, 2016). SLA has drawn more attention from researchers because the success of L2 learners is wide-ranging. The differential success was caused by various factors, which often were due to age constraints related with SLA, which is absent or not as eminent in the development of a first or native language. Age having an influence on SLA is an undeniable fact, and studies regarding age-related issues in SLA is broadly divided into two types: the existence and characteristics of maturational constraints and identifying Age of Acquisition (AoA), and differences in second or foreign language learning (Muñoz, 2008, p. 578). Therefore, a discussion regarding effect of age in SLA from the perspectives of CPH and UA is necessary.

As mentioned above, the two aspects, age of acquisition and differences in second language learning correlate with each other and reasonably comprise the hypothesis of age effect in SLA. The effect of age in SLA is encircled by the notion of Critical Period Hypothesis (CPH); therefore, a comparison to Ultimate Attainment (UA) is particularly relevant regarding $\mathrm{CPH}$ since UA is often considered to be the determining factor to identify successful SLA learners. In literature, the discussion of Ultimate Attainment or proficiency like a native speaker is closely related with $\mathrm{CPH}$, as the prior claim behind this is that exposure to a second language from a very early age often can result in proficiency like a native speaker. Perhaps, it is true that children can attain a native-like proficiency without much striving, while adults study hard and diligently for years with unsatisfactory results $(\mathrm{Li}, 2015)$. Moreover, besides having an accent or certain pronunciations, many researchers oppose the advantage of being young learners and claim that older learners are more effective learners when they get to be in a linguistics environment and have language input (Ellis, 1994). As a result, the existence of CPH cannot be proved entirely and argued widely. On the contrary, UA must not be the determining factor to evaluate a SL achievement. Despite the evident differences between L1 and L2 learners, considering the endless exposure of learning in L1, how far L2 learner barriers in picking up a language with minimal input and socio-psychological issues in individual learners can be compared with L1 learners is open to question. 
Besides theoretical issues, few technical issues are related in this inevitable problem to be solved. In the field of SLA, more specifically, age-related studies lack uniformity and methodological soundness (Muñoz \& Singleton, 2011; DeKeyser et al., 2010). Due to this, the available findings in literature is inconclusive and speculative. Therefore, the question of age in SLA is critical to be answered and has immense significance to the advancement of the field. Therefore, before reaching any conclusion in this regard, it would be wise to review exiting empirical studies to find more in-depth information of the phenomenon.

\subsection{Aim of the Study}

The study investigates the role of AoA in Second Language Acquisition, in relation to UA as a point of comparison and the inconclusiveness of research findings in this regard. Recent literature figured prominently these scopes according to the aim of the research:

- To scrutinise what extent to which we can speak of a genuinely unitary Critical Period Hypothesis (CPH);

- To explore whether it is relevant to compare L2 attainment in Critical Period Hypothesis (CPH) to Ultimate Attainment or native proficiency.

- To inspect research aspects that created diversity in research findings and future implications for Age of Acquisition (AoA) studies in SLA.

\subsection{Significance of the Study}

The age-related study in SLA is significant for many reasons, different people want to know about the process for different self-interest. For instance, the psychologists Monner, Vatz, Morini, Hwang, \& DeKeyser (2012) perceive that age-related questions unavoidably lead to reflection on the basic nature of possible learning processes of language. On the other hand, for teachers and educators, these questions are of very important concern in pedagogy and curriculum or material development for different ages of learners. Even learners who started learning or receiving language input, often have found to end up without UA in their target language (Christie, 2012). Therefore, the present study can open new avenues for all who are associated with teaching learning and researching second language teaching and learning.

\section{Methodology}

Based on the aim of the study, the present study chose a systematic review process and reviewed extensive literature on the effect of age in SLA in relation with the CPH (Critical Period Hypothesis) and UA (Ultimate Attainment) in a second language. Secondary data analysis, as described by Boslaugh (2007), "In the broadest sense, analysis of data collected by someone else" (p. 9). Secondary data analysis has been growing in popularity and was adopted by many researchers in their studies to discuss issues in SLA and other issues in linguistics (See- Ellis, 2010; DeKeyser, 2013; Karim \& Rahman, 2016).

The conduct of a systematic review depends heavily on the scope and quality of the included studies. Thus, systematic reviewers may need to modify their original review protocol during its conduct (Moher, Liberati, Tetzlaff, Altman, \& Prisma Group, 2009). In order to search peer-reviewed articles, a systematic search was conducted in SCOPUS and Google Scholar for the lasy fifteen years (2000-present), with relevant keywords, such as Age effect on SLA, Critical Period Hypothesis (CPH), and Ultimate Attainment (UA). Some of the articles had to be excluded due to their irrelevance. In the search of SCOPUS, ten relevant articles were found to be specific to our topic. In the Google Scholar search, 15 articles were found to fit to our topic.

In analysis, the paper firstly analysed the $\mathrm{CPH}$ and the issue of age in language attainment; secondly, the issue of Ultimate Attainment in Language Learning from the perspective of $\mathrm{CPH}$ was discussed. The discussion was put in a thematic manner based on the related variables of the study and will be allowed to discuss the phenomenon in a structural way.

\section{Critical Period Hypothesis and SLA}

Penfield \& Roberts (1959), two neurobiologists, first put forward the notion of the critical period hypothesis, which was primarily derived from biology. The Critical Period Hypothesis refers to a particular time of human life that allows people to acquire a language in a natural environment faster and easier without any outside intervention and formal instruction (Fromkin, Rodman, \& Hyams, 1983). Later, a theory called, Biological Foundations of Language, developed by Lenneberg (1967), suggested that natural language acquisition "by mere exposure" could only take place during a critical period, lasting from about age two to puberty.

The conclusion that Penfield and Roberts made was that after ninth year or Critical Period, the human brain becomes progressively stiff and rigid for learning languages (Singleton, 2005). Contrastingly, Lenneberg (1967), suggested puberty was the offset point for the critical period and further explained: "the incidence of 
"language-learning-blocks" rapidly increases", "foreign languages have to be learned through a conscious and laboured effort", and "[f]oreign accents cannot be overcome easily" (p. 176). Nevertheless, the bottom-line is that if the hypothesis is true, then the Critical Period Hypothesis (CPH) must have a crucial role to play in the attainment of a second language as well.

However, in the existing literature on SLA, it was found to be alienated among the propositions of effect of age and the capacity of second language acquisition by humans in relation to the critical period hypothesis, whether there is any association of Critical Period (CP) ending due to the acquisitions of an additional language, or if there are any qualitative differences with late language acquisition. To sum up a proposition, Miralpeix (2011) described the relation to a possible Critical Period in SLA: (1) "the younger, the better", (2) "the older, the better", (3) "the younger, the better in some respects", (4) "the younger, the better in the long run" and (5) the "qualitative change" position, which claims that after a certain age, qualitative changes occur in the learning process.

The first line of the research is related to the question about the existence of the Critical Period Hypothesis. The study's findings have proven to be controversial since the experts made no determination regarding the $\mathrm{CPH}$ and second language acquisition. Describing the significance of $\mathrm{CPH}$ in the attainment of a Second Language, Hyltenstam \& Abrahamsson (2000, p. 155) assert that no post-CPH L2 learner has thus far reached the proficiency level of a native speaker. Even the very young learners of a second language differ from native speakers, regarding their level of competencies of the lexico-grammatical dimension (Hyltenstam \& Abrahamsson, 2000). Moreover, the same notion is applicable in the development of second language pronunciation as well. According to Flege (2002), even though individuals are exposed to an L2, in an L2 environment, young children often end up speaking the L2 with a non-native accent. The above-mentioned notion was well backed by a study by McDonald (2000), in which it was found that early exposure of English from a Spanish-speaking background had clear advantages on the grammatical judgment test and were able to perform to native levels.

Native language and L1 Cultural identity (linguistico-cultural) is closely associated with the discussion of CPH, since it was found in the study that people who immigrate after 10 years of age often tend to be dominated by their own culture and language; however, the opposite result was found in the case of immigrants who are under-10 years old, they often switch their linguistico-cultural identity and transform their dominant language to the language and culture of the host country (Jia \& Aaronson, 2003).

Contrastingly, numerous studies failed to correlate between age of acquisition and ultimate attainment positively. Moreover, many more researchers accept the negative correlation between age and Critical Period Hypothesis as a fact (DeKeyser, Shabtay \& Ravid, 2010). However, Ellis \& Sagarra (2010) argued that there is limited attainment among adult learners of a second language. The claim was further attained by the study of Hakuta, Bialystok \& Wiley (2003), where they established a negative correlation between age and SLA, and found no advantage among those of a young age with the attainment of a second language.

In summary, the notion of CPH in SLA is still a topic of debate amid researchers. Whether it exists or if it existed, then which aspects of language acquisition are influenced by $\mathrm{CPH}$, other than phonological acquisition, is a subject of further research.

\section{UA is the Point of Comparison}

SLA researchers were found to be slightly partial to compare ultimate attainment as the point of comparison in SLA. Ultimate attainment refers to native-like proficiency of a language. The CPH and UA debate was raised due to previous studies being conducted where many researchers expressed the view that after a certain age, L2 learners cannot possess complete acquisition. Long (2007) reported the notion in a more general regard that L2 learners cannot acquire different skills, such as phonetics, grammar, vocabulary, etc. in comparison to native speakers. However, the notion was challenged by numerous researchers, e.g., Bongaerts (2003); Muñoz \& Singleton (2007); Kinsella (2009); Muñoz \& David Singleton (2011), who proved that late beginners also possess L2 proficiency.

Building up the argument that existing literature is already being debated lets us consider that $\mathrm{CPH}$ is existent. However, to what extent it is justified to compare an L2 achiever to a native speaker's level of proficiency is debatable. The phenomenon was explained in Cenoz \& Genesee (1998, p. 18), "bilinguals, in and outside the school, are usually evaluated against "monolingual" competence in their non-native languages." It is a pessimistic and exaggeratedly premature approach to evaluate the achievement of a non-native speaker, especially to a learner who is learning the language in an instructional setting, who are adult learners in general. 
Of particular criticism of such a comparison would be the relationship between language and culture. Native language is highly culture bound (Hill, 1970). Therefore, in a multicultural society like India, where people are mostly multilingual, they cannot be counted as native speakers for L2 or L3. In particular, a typical South Indian may speak and understand Hindi, Marathi, or Panjabi but, cannot be a native speaker since at some point of time, their culture will interfere.

The idea was even found irrational for late L1 acquirers. Late acquisition can be caused by different reasons, often psychological, but it also can have external reasons. If a child experiences negligence in their first five years, they often end up facing complexities, such as permanently smaller head circumference, smaller brain size, and impaired ability to learn language as well as developing normal social behaviour (Muñoz \& Singleton, 2011). Therefore, it would be an equally exclusive approach to evaluate their acquisition to UA of language.

Among many others is Cook (2002), who argues that L2 users should be evaluated in their own rights and standards, rather than an unequal comparison with native speakers. According to him, "ultimate attainment is a monolingual standard rather than an L2 standard" (2002, p. 6). The comparison is impossible to make biologically as well. In L1 and L2, the representation is different in our brain. In a sense similar to Chomsky, the associated mental representations, as Rothman puts it:

Any version of the $\mathrm{CPH}$ that claims that adults lose the ability to acquire syntax, for example, in the way children do, is forced to take the position that apparent L2 knowledge of target syntactic properties absent from or different in the L1 were learned explicitly and are therefore represented differently in the brain. (Rothman, 2008, p. 1065)

The literature is mostly inclined to the notion that late learners also can be successful learners. Therefore, $\mathrm{CPH}$ should not be correlated with UA anymore. However, the above discussion also points out the unfair comparison achievement between L2 achievements with native-like proficiency. Therefore, whether the CPH is available or not can be argued, however, the point of comparison should not be UA or native-like proficiency, which is beyond argument.

\section{Contextual and Intrapersonal Contribution}

Empirical studies in SLA depend on contextual and intrapersonal differences. For instance, in the empirical basis of skill acquisition theory (DeKeyser, 1997), the results were found to be inconclusive in different contexts and not transferable to other contexts (Maftoon, Shakouri, \& Nazari, 2013), among other inconclusive examples. In this study, the factors that contributed towards overall achievement were: amount of L2 input and socio-psychological differences.

L2 Input and Achievement. The measurement of input, rather than the length of residence, exposure to language, and quality of input were supposed to be the testing criteria. A study by Jia \& Aaronson (2003) pointed out the differences between level of achievement for the same LoR based on the difference in exposure and the input of the language. Achievement was found to be higher among the L1 users who were exposed to the target language. It is relatively true in the case of instructed input, e.g., learning L2 in a classroom setting for a period of time and using the language outside according to Muñoz and David Singleton (2011). Saito \& Brajot (2013) in their study, had shown that length of residence (LoR) and Length of Instruction (LoI) have an immense effect on the acquisition of $\mathrm{L} 2$ attainment.

Socio-Psychological differences. Learners through socio-affective and cognitive factors have proven impact on achievement of L2 performance. In their empirical study on second language vocabulary acquisition, Nosratinia, Abbasi, \& Zaker (2015) established a relationship between critical thinking, autonomy, and vocabulary, which are entirely intrapersonal attributes that varies from individual to individual. Distinctly from the psychological attributes that social factors have, Moyer (2004), as cited in Muñoz and Singleton (2011), articulated that socio-psychological factors through qualitative analysis of interview data underscored a set of psychological and social influences in learners' individual learning experience: opportunities for contact, attitudes toward the target language culture, sense of self in L2 (motivation, behaviour and language function), perceptions of foreignness, and belonging.

Studies conducted in the field of SLA indicates that L2 learning is not only something that happens automatically, but different intra and external factors are associated with it. These variables are subject to future research to draw further conclusions on the age effect in SLA.

\section{CPH: Inconclusive Research}

Despite the enormous amount of research that was conducted, advancement is meagre. The reasons behind ultimate attainment and $\mathrm{CPH}$ being an unsolved mystery laying behind the shade of poor methodological 
instrumentation (DeKeyser et al., 2010).

The dominance of quantitative approach to SLA research was one of the most significant challenges in this regard. Hulstijn (2014), cited in Rahman \& Pandian (2016), articulated the distinct nature between positivism and interpretivism, which can differentiate one research from another. The ultimate attainment were mostly evaluated in the numbers by grammatical judgement tests with a few variables (DeKeyser et al., 2010). Moreover, present-day SLA research is also dominated by lab-based experiments, which are fundamentally wrong for the conception of research in SLA since it eliminates the natural setting of research at the start (Rahman \& Pandian, 2016; Light \& Gnida, 2012).

The questionable sampling is often attributed to SLA research, more precisely, research related to age of acquisition, $\mathrm{CPH}$ and UA. The sample is unsystematic and examined in an unstructured manner. The key challenge in this regard is the likelihood of different puberty periods in different individuals. Cognitive/psychological development is often found to be varied from person to person, which has a tremendous impact on research outcome. Schumann (1995) argued for the reasoning, explanation, and narrative aspects of language acquisition over the evaluation of linguistic accuracy tests. Therefore, in the meta-phonological sphere, which means that research on morphology and syntax should be given priority to rise beyond the notion of only linguistic competence in SLA.

\section{Implication of the Findings}

The present study aimed to address three key issues in the age-related debate. Based on the discussion in the present article, the findings have four implications. Firstly, whether the effect of age in SLA should be debated or not and what will be following consequences; secondly, whether it is justified to compare a second language learner's achievement to UA to inspect the effect of age in SLA; thirdly, the issues that need to be considered in the research in SLA to bring uniformity in the field.

The relationship of age and SLA needs to be resolved and undeniably, the results have a wide range of implications in second or foreign language teaching/learning, language policy and planning. The fallacy is still evident based on $\mathrm{CPH}$, which asserts an earlier start is better in terms of language learning, which was challenged by scholars in the field, such as DeKeyser, Shabtay, \& Ravid (2010). In the present study as well, the notion is criticised critically based on empirical studies. However, in a hypothetical state, we may argue that in terms of acquiring native-like pronunciation, an early start may give some advantages, nevertheless, in terms of other aspects of acquisition, any positive correlation of age was not found. If the belief holds true, the hegemony in the planning of English language policy in a second or foreign language context suggests that early introduction of a second language access needs to be re-evaluated entirely. Thus in consequence, English language teaching methodology, curriculum, material, teachers' beliefs, and practice will face radical changes.

The point of departure to assess a successful or unsuccessful learner should not be ultimate attainment and according to the discussion above, it is also unfair. It can demotivate learners who are giving effort for a long time period to learn a language. In line with earlier studies, Rothman (2008) presented a study that strengthens the challenged view that says the ultimate attainment for monolingual speakers, second language learners should have separate evaluating criteria and assessment systems. This implies worldwide language testing systems like IELTS, TOEFL, and PTE, etc. would require developing a new set of criteria that will focus second language learner characteristics that are unique to them. They will be assessed based on their counterparts, second language learners.

Despite the large amount of SLA research that was conducted in the field, qualitative and quantitative, the advancement is few. The present study pointed out the ununiformed research and lack of methodological clarity and creativity. The study was introduced by saying that, the age-related debate is fundamentally posed on CPH and UA, at least that is what the literature advises. However, what most of the studies missed out in the debate is that they have overlooked the widely-neglected importance of quality, amount of input, as well as learners' attitudes and orientations that were discussed in the contextual factors and intrapersonal contribution. Contextual constraints are inevitable in SLA, since quality and amount of input that learners receive is discussed in the study and the findings are in line with earlier studies, such as Maftoon, Shakouri, \& Nazari (2013). Muñoz \& Singleton (2011) highlighted contextual factors and intrapersonal contribution as the important variables to consider in future studies to strengthen the findings. The research among the adult language learners should consider other variables while researching. Personality, motivation, and level of education, among other variables, according to Dornyei (2005) and DeKeyser et al. (2010), these are most important characteristics to consider since these aspects have a significant impact on learning or acquiring L2, as issues related to age have shown.

It is necessitated for future studies to be more articulated in selecting dependent variables. In CPH and UA, the 
dependent variables are further conceptualised, however, the challenge of selecting a dependable variable is still not coherent. Using the same grammatical judgement instrument, DeKeyser et al. (2010) questioned the credibility of grammar achievement and L2 achievement. Research variables and instruments should be revised for longitudinal research, in conjunction with the nature of research to ensure that development in SLA can be noted based on the different phases in relation with different independent and dependent variables.

\section{References}

Bongaerts, T. (2003). Effets de l'âge sur l'acquisition de la prononciation d'une seconde langue. Acquisition et interaction en langue étrangère, (18), 79-98.

Boslaugh, S. (2007). Secondary data sources for public health: A practical guide. Cambridge: Cambridge University Press. https://doi.org/10.1017/CBO9780511618802

Cenoz, J., \& Genesee, F. (1998). Psycholinguistic perspectives on multilingualism and multilingual education. In J. Cenoz \& F. Genesee (Eds.), Beyond bilingualism: Multilingualism and multilingual education (pp. 16-32). Clevedon, Multilingual Matters.

Christie, F. (2012). Language education throughout the school years: A functional perspective. Language Learning, 62(Supplement 1), 1-247. Malden, MA: Wiley-Blackwell.

Cook, V. (Ed.) (2002). Portraits of the L2 user. Clevedon: Multilingual Matters Ltd.

DeKeyser, R. M. (2007). Skill acquisition theory. In B. VanPatten \& J. Williams (Eds.), Theories in second language acquisition (pp. 97-113). London: Lawrence Erlbaum Associates

DeKeyser, R. M. (2013). Age effects in second language learning: Stepping stones toward better understanding. Language Learning, 63(s1), 52-67. https://doi.org/10.1111/j.1467-9922.2012.00737.x

DeKeyser, R., Alfi-Shabtay, I., \& Ravid, D. (2010). Cross-linguistic evidence for the nature of age effects in second language acquisition. Applied Psycholinguistics, 31(03), 413-438. https://doi.org/10.1017/S0142716410000056

Ellis, N. C., \& Sagarra, N. (2010). The bounds of adult language acquisition. Studies in Second Language Acquisition, 32(04), 553-580. https://doi.org/10.1017/S0272263110000264

Ellis, R. (1994). The study of second language acquisition. Oxford University.

Flege, J. E. (2002). Interactions between the native and second-language phonetic systems. In T. P. Burmeister, $\&$ A. Rohde (Eds.), An integrated view of language development: Papers in honor of Henning Wode (pp. 217-244). Trier: Wissenschaftlicher Verlag.

Hakuta, K., Bialystok, E., \& Wiley, E. (2003). Critical evidence a test of the critical-period hypothesis for second-language acquisition. Psychological Science, 14(1), 31-38. https://doi.org/10.1111/1467-9280.01415

Hill, J. (1970). Foreign accents, language acquisition and cerebral dominance revisited. Language Learning, 20(2), 237-248. https://doi.org/10.1111/j.1467-1770.1970.tb00480.x

Hyltenstam, K., \& Abrahamsson, N. (2000). Who can become native-like in a second language? All, some, or none?. Studia linguistica, 54(2), 150-166. https://doi.org/10.1111/1467-9582.00056

Jia, G., \& Aaronson, D. (2003). A longitudinal study of Chinese children and adolescents learning English in the United States. Applied Psycholinguistics, 24, 131-161. https://doi.org/10.1017/S0142716403000079

Kinsella, C. (2009). An investigation into the proficiency of successful late learners of French. Ph.D. dissertation. Trinity College Dublin.

Lenneberg, E. H. (1967). Biological foundations of language. New York: Wiley.

Li, Z. H. O. U. (2015). Can Adults Attain a Native-Like Accent in Their Second Language? Sino-US English Teaching, 12(6), 403-409.

Light, J., \& Gnida, S. (2012). Mind the Gap: How a Project in Alberta Attempted to Narrow the Gap between Classroom Teachers and Language Education Research. TESL Canada Journal, 30(1), 139-150. https://doi.org/10.18806/tesl.v30i1.1131

Long, M. H. (2007). Problems in SLA. Mahwah, NJ: Lawrence Erlbaum Associates.

Maftoon, P., Shakouri, N., \& Nazari, O. (2013). On Situating the Stance of Practice in SLA. International Journal of Applied Linguistics and English Literature, 2(2), 71-76. https://doi.org/10.7575/aiac.ijalel.v.2n.2p.71 
McDonald, J. L. (2000). Grammaticality judgments in a second language: Influences of age of acquisition and native language. Applied Psycholinguistics, 21, 395-423. https://doi.org/10.1017/S0142716400003064

Miralpeix, I. (2011). Age effects on second language acquisition: Critical issues under debate. In D. Birdsong (Ed.), Second language acquisition and the Critical Period Hypothesis. Language Teaching, 44(01), 122-132.

Moher, D., Liberati, A., Tetzlaff, J., Altman, D. G., \& Prisma Group. (2009). Preferred reporting items for systematic reviews and meta-analyses: the PRISMA statement. PLoS med, 6(7), e1000097. https://doi.org/10.1371/journal.pmed.1000097

Monner, D., Vatz, K., Morini, G., Hwang, S. O., \& DeKeyser, R. M. (2012). A neural network model of the effects of entrenchment and memory development on grammatical gender learning. Bilingualism: Language and Cognition.

Mũnoz, C., \& Singleton, D. (2007). Foreign accent in advanced learners: two successful profiles. The EUROSLA Yearbook, 7, 171-190. https://doi.org/10.1075/eurosla.7.10mun

Muñoz, C. (2008). Symmetries and asymmetries of age effects in naturalistic and instructed L2 learning. Applied Linguistics, 29(4), 578-596. https://doi.org/10.1093/applin/amm056

Muñoz, C., \& Singleton, D. (2011). A critical review of age-related research on L2 ultimate attainment. Language Teaching, 44(1), 1-35. https://doi.org/10.1017/S0261444810000327

Nosratinia, M., Abbasi, M., \& Zaker, A. (2015). Promoting Second Language Learners' Vocabulary Learning Strategies: Can Autonomy and Critical Thinking Make a Contribution? International Journal of Applied Linguistics and English Literature, 4(3), 21-30.

Penfield, W., \& Roberts, L. (1959). Speech and brain mechanisms. Princeton: Princeton University Press.

Rahman, M. M., \& Pandian, A. (2016). The Gap between Research and Practice in the Field of SLA: The Rationale behind It and Bridging the Gap. Journal of Applied Linguistics and Language Research, 3(3), $162-172$.

Rothman, J. (2008). Why all counter-evidence to the Critical Period Hypothesis is not equal or problematic. Language and Linguistics Compass, 2(6), 1063-1088. https://doi.org/10.1111/j.1749-818X.2008.00098.x

Schumann, J. H. (1995). Ad minorem theoriae gloriam. Studies in Second Language Acquisition, 17, 59-65. https://doi.org/10.1017/S0272263100013759

Singleton, D. (2005). The critical period hypothesis: A coat of many colours. International Review of Applied Linguistics in Language Teaching, 43, 269-285. https://doi.org/10.1515/iral.2005.43.4.269

\section{Copyrights}

Copyright for this article is retained by the author(s), with first publication rights granted to the journal.

This is an open-access article distributed under the terms and conditions of the Creative Commons Attribution license (http://creativecommons.org/licenses/by/4.0/). 